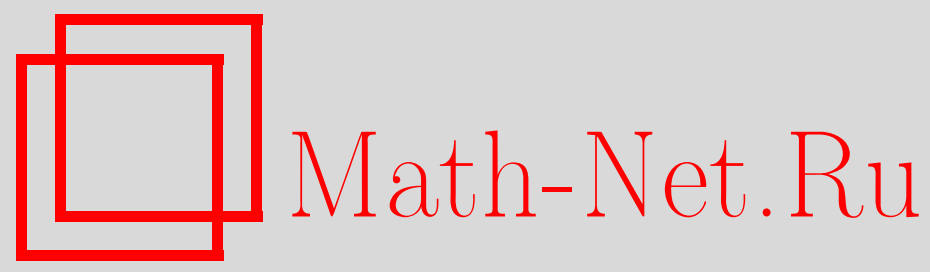

Д. В. Миллионщиков, Когомологии разрешимых алгебр Ли и солвмногообразия, Матем. заметки, 2005, том 77, выпуск 1, 67-79

DOI: https://doi.org/10.4213/mzm2470

Использование Общероссийского математического портала Math-Net.Ru подразумевает, что вы прочитали и согласны с пользовательским соглашением http://www.mathnet.ru/rus/agreement

Параметры загрузки:

IP : 54.89 .56 .158

26 апреля 2023 г., $17: 18: 56$

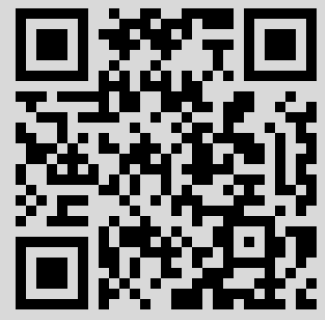




\section{КОГОМОЛОГИИ РАЗРЕШИМЫХ АЛГЕБР ЛИ И СОЛВМНОГООБРАЗИЯ}

\section{Д. В. Миллионщиков}

В работе изучаются когомологии $H_{\lambda \omega}^{*}(G / \Gamma, \mathbb{C})$ комплекса де Рама $\Lambda^{*}(G / \Gamma) \otimes \mathbb{C}$ компактного солвмногообразия $G / \Gamma$ с деформированньм дифференциалом $d_{\lambda \omega}=d+$ $\lambda \omega$, где $\omega$ - некоторая замкнутая 1-форма. Такие когомологии естественным образом возникают в теории Морса-Новикова. Показывается, что для произвольной вполне разрешшмой группы Ли $G$ с кокомпактной решеткой $\Gamma \subset G$ когомологии $H_{\lambda \omega}^{*}(G / \Gamma, \mathbb{C})$ изоморфны когомологиям $H_{\lambda \omega}^{*}(\mathfrak{g})$ касательной алгебры Ли $\mathfrak{g}$ группы $G$ с коэффициентами в одномерном представлении $\rho_{\lambda \omega}: \mathfrak{g} \rightarrow \mathbb{K}, \rho_{\lambda \omega}(\xi)=\lambda \omega(\xi)$. Кроме того, когомологии $H_{\lambda \omega}^{*}(G / \Gamma, \mathbb{C})$ нетривиальны тогда и только тогда, когда $-\lambda[\omega]$ принадлежит конечному подмножеству $\widetilde{\Omega}_{\mathfrak{g}}$ в $H^{1}(G / \Gamma, \mathbb{C})$, определенному в терминах алгебры Ли $\mathfrak{g}$.

Библиографоия: 17 названий.

1. Введение. В начале 80-х годов С.П. Новиков построил [1], [2] аналог теории Морса для гладких замкнутых 1-форм на компактном гладком многообразии $M$. В частности, им были предложены неравенства типа Морса (неравенства Новикова) для чисел $m_{p}(\omega)$ нулей индекса $p$ произвольной гладкой замкнутой морсовской 1-формы $\omega$ на M. В работах [3], [4] был предложен метод для нахождения так назьваемых неравенств Новикова без кручения в терминах комплекса де Рама многообразия $M$. Этот метод основывался на подходе Виттена [5] к теории Морса. А. В. Пажитнов получил ряд важных результатов в этом направлении в [4]. Когомологии комплекса $\Lambda^{*}(M) \otimes \mathbb{C}$ с деформированньм дифференциалом $d+\lambda \omega$ изоморфны когомологиям $H_{\rho_{\lambda \omega}}^{*}(M, \mathbb{C})$ многообразия $M$ с коэффициентами в локальной системе $\rho_{\lambda \omega}$ груш $\mathbb{C}, \rho_{\lambda \omega}(\gamma)=\exp \int_{\gamma} \lambda \omega, \gamma \in \pi_{1}(M)$, и для достаточно больших вещественных чисел $\lambda$ была получена следующая оценка [4]:

$$
m_{p}(\omega) \geqslant \operatorname{dim} H_{\rho_{\lambda \omega}}^{p}(M, \mathbb{C}) \quad \forall p
$$

Л.А. Алания в [6] рассмотрел когомологии $H_{\rho_{\lambda \omega}}^{*}\left(M_{n}, \mathbb{C}\right)$ одной серии нильмногообразий $M_{n}$. Он доказал, что $H_{\rho_{\lambda \omega}}^{*}\left(M_{n}, \mathbb{C}\right)$ являются тривиальными тогда и только тогда, когда $\lambda \omega \neq 0$. Случай $\lambda \omega=0$ рассматривался в [7]. В обоих случаях доказательство было основано на теореме Номидзу [8] и все когомологическиевычисления проводились в терминах соответствующей нильпотентной алгебры Ли $\mathscr{V}_{n}$.

Работа выполнена при частичной поддержке Российского фонда фундаментальных исследований, грант № 02-01-00659, гранта PAI-Pоссия № 04495UL и гранта № HШ-2185.2003.1. 
Отправной точкой данной работы стало стремление:

а) распространить результаты [6] в более общей ситуации, рассматривая солвмногообразия;

б) найти простые примеры многообразий с нетривиальньми $H_{\rho_{\lambda \omega}^{*}}^{*}(M, \mathbb{C})$ с $\lambda \omega \neq 0$. Одним из первых наблюдений, сделанных в этом направлении, стало следующее утверждение: для произвольного нильмногообразия $G / \Gamma$ когомологии $H_{\lambda \omega}^{*}(G / \Gamma, \mathbb{C})$ изоморфны когомологиям алгебры Ли $H_{\lambda \omega}^{*}(\mathfrak{g})$ с коэффичиентами в одномерном представлении $\rho_{\lambda \omega}: \mathfrak{g} \rightarrow \mathbb{C}, \rho_{\lambda \omega}(\xi)=\lambda \omega(\xi)$, и тем самьг, $H_{\lambda \omega}^{*}(\mathfrak{g})=0$ в силу теоремы Диксмье [9] (следствие 2.3).

Используя точную последовательность Диксмье когомологий алгебр Ли, показано, что для произвольной алгебры Ли $\mathfrak{g} c H^{1}(\mathfrak{g}) \neq 0$ на каждой прямой $\lambda \omega \subset H^{1}(\mathfrak{g})$ существует лиш конечное число значений параметра $\lambda_{i}$, для которых когомологии $H_{\lambda_{i} \omega}^{*}(\mathfrak{g})$ нетривиальны (следствие 2.1).

Используя теорему Хаттори [10], можно показать, что изоморфизм $H_{\lambda \omega}^{*}(G / \Gamma, \mathbb{C}) \cong$ $H_{\lambda \omega}^{*}(\mathfrak{g})$ сохраняется и для компактных солвмногообразий $G / \Gamma$ с вполне разрешимой группой $G$. С другой стороны, для вполне разрешимой алгебры Ли g можно так подобрать базис в $\mathfrak{g}^{*}$, что

$$
d \omega_{i}=0, \quad i=1, \ldots, k, \quad d \omega_{j}=\alpha_{j} \wedge \omega_{j}+P_{j}\left(\omega_{1}, \ldots, \omega_{j-1}\right), \quad j=k+1, \ldots, n .
$$

С помощь такого базиса определяется естественная фильтрация коцепного комплекса $\left(\Lambda^{*}\left(\mathfrak{g}^{*}\right), d_{\lambda \omega}\right)$ и соответствующая спектральная последовательность $E_{r}$, сходящаяся к $H_{\lambda \omega}^{*}(\mathfrak{g}) . E_{r}$ вырождается в первом члене $E_{1}$ почти всегда, за исключением случая, когда $-\lambda \omega$ принадлежит конечному подмножеству $\Omega_{\mathfrak{g}} \subset H^{1}(\mathfrak{g})$ - множеству сумм замкнутых 1-форм из набора $\left\{\alpha_{j}\right\}$ весов вполне приводимого представления, ассоциированного с присоединенным представлением ad (теорема 3.1 ).

Основной результат настоящей работы (теорема 4.5): когомологии с локальными коэффициентами $H_{\lambda \omega}^{*}(G / \Gamma, \mathbb{C})$ компактного солвмногообразия $G / \Gamma$, где $G$-вполне разрешимая группа Ли, являются нетривиальнылми тогда и только тогда, когда $-\lambda[\omega] \in \widetilde{\Omega}_{\mathfrak{g}}$, где $\widetilde{\Omega}_{\mathfrak{g}}$ является конечным подмножеством в $H^{1}(G / \Gamma, \mathbb{C})$, корректно определенным в терминах алгебры Ли g.

Часть результатов была анонсирована в [11]. Автор благодарен Л. А. Алания за плодотворные обсуждения и внимание к работе.

\section{1. Когомологии с локальными коэффициентами, деформированный комп-} лекс де Рама и теория Морса-Новикова. Рассмотрим замкнутоекомпактноемногообразие $M$, его фундаментальную группу $\pi_{1}(M)=\Gamma$, действующую справа на универсальном накрытии $p: \widetilde{M} \rightarrow M$. Пусть $\rho: \Gamma \rightarrow \mathrm{GL}(F)$ - представление Г в группе автоморфизмов некоторой абелевой группы $F$. В этом случае можно определить правое действие $Г$ на прямом произведении $\widetilde{M} \times F$ :

$$
(x, u) \gamma=\left(x \gamma, \rho(\gamma)^{-1}(u)\right), \quad x \in \widetilde{M}, \quad u \in F, \quad \gamma \in \Gamma
$$


ОПРЕДЕЛЕНИЕ 1.1 (Стинрод [12]). Локальной системой $L_{\rho}$ групा с группой $F$ в качестве типичного слоя назьвается факторпространство $(\widetilde{M} \times F) / \Gamma$.

В данной работе мы будем рассматривать случай $F=\mathbb{C}$. Локальная система $L_{\rho}$ будет в этом случае одномерным векторным расслоением над $M$ или, в других терминах, локально постоянным одномерным пучком над $M$.

У каждой точки $x \in M$ есть своя группа коэффициентов $F_{x}$, изоморфная $F$. Рассматривая $M$ как клеточный комплекс $K_{M}$ и выбрав в каждой клетке $\sigma \in K_{M}$ по точке $x_{\sigma}$, будем считать, что у клетки $\sigma$ задана своя группа коэффициентов $F_{\sigma}=F_{x_{\sigma}}$. Зафиксируем некоторую точку $x_{\sigma_{0}} \in K_{M}$ и систему путей $\left\{\gamma_{\tau \sigma}\right\}$, соединяющих между собой все точки вида $x_{\sigma}$. Каждому ориентированному пути $\gamma_{\sigma \sigma_{0}}$ из точки $x_{\sigma}$ в точку $x_{\sigma_{0}}$ сопоставим изоморфизм $w_{\sigma_{0} \sigma}: F_{\sigma_{0}} \rightarrow F_{\sigma}$. Рассмотрим теперь две различные клетки $\sigma$ и $\tau$. Петля $\gamma=\gamma_{\sigma \sigma_{0}}^{-1} \gamma_{\sigma \tau} \gamma_{\tau \sigma_{0}}$ определяет элемент $\gamma \in \Gamma$, а значит, и автоморфизм $\rho(\gamma): F_{\sigma_{0}} \rightarrow F_{\sigma_{0}}$. Таким образом, определен изоморфизм

$$
w_{\sigma \tau}: F_{\sigma} \rightarrow F_{\tau}, \quad w_{\sigma \tau}=w_{\tau \sigma_{0}}^{-1} \rho(\gamma) w_{\sigma \sigma_{0}} .
$$

Определим теперь коцепной комплекс $\left(C_{\rho}^{*}\left(K_{M}\right), \delta_{\rho}\right)$ с коэффициентами в локальной системе $L_{\rho}$. Коцепь $c \in C_{\rho}^{p}\left(K_{M}\right)$ определим как линейную функцию, сопоставляющую каждой (ориентированной) $p$-клетке $\sigma$ элемент $c(\sigma) \in F_{\sigma}$. Кограничный оператор $\delta_{\rho}$ определяется соответственно:

$$
\delta_{\rho} c(\tau)=\sum[\sigma: \tau] w_{\sigma \tau}(c(\sigma)),
$$

где целое число $[\sigma: \tau]$ обозначает коэффициент инцидентности между $\sigma$ и $\tau$, а суммирование ведется по всем клеткам $\sigma$ на единицу меньшей размерности, входящим в границу $\partial \tau$ клетки $\tau$.

ОПРЕДЕЛЕНИЕ 1.2 (Стинрод [12]). Когомологиями $H_{\rho}^{*}\left(K_{M}, F\right)$ клеточного комплекса $K_{M}$ (многообразия $M$ ) с коэффициентами в локальной системе $L_{\rho}$ называются когомологии коцепного комплекса $\left(C_{\rho}^{*}(K), \delta_{\rho}\right)$.

Рассмотрим комплекс де Рама $\left(\Lambda^{*}(M), d\right)$ многообразия $M$. Пусть $\omega$ - некоторая замкнутая 1-форма на многообразии $M$ и $\lambda \in \mathbb{R}$. С помошью $\omega$ можно определить представление фундаментальной групшы $\rho_{\lambda \omega}: \pi_{1}(M) \rightarrow \mathrm{GL}(\mathbb{C})=\mathbb{C}^{*}:$

$$
\rho_{\lambda \omega}(\gamma)=\exp \int_{\gamma} \lambda \omega, \quad \gamma \in \pi_{1}(M) .
$$

С другой стороны, при помощи $\omega$ определяется комплекс де Рама $\left(\Lambda^{*}(M), d_{\lambda \omega}\right)$ с деформированньм дифференциалом

$$
d_{\lambda \omega}=d+\lambda \omega: \Lambda^{*}(M) \rightarrow \Lambda^{*}(M),
$$

т.е. для произвольной формы $a \in \Lambda^{*}(M)$ имеем

$$
d_{\lambda \omega}(a)=d a+\lambda \omega \wedge a .
$$

Выбрав $\lambda \in \mathbb{C}$ и рассматривая комплексификацию $\Lambda^{*}(M) \otimes \mathbb{C}$, приходим к следующему утверждению. 
Лемма 1.1 [3], [4]. 1) Для произвольной замкнутой 1-формы $d \omega=0$ когомологии $H_{\lambda \omega}^{*}(M, \mathbb{C})$ алгебраического комплекса $\left(\Lambda^{*}(M) \otimes \mathbb{C}, d_{\lambda \omega}\right)$ изоморфны когомологиям $H_{\rho_{\lambda \omega}^{*}}^{*}(M, \mathbb{C})$ с коәффичиентами в локальной системе әрупп $\mathbb{C}$, заданной представлением (1) фундаментальной группы $\rho_{\lambda \omega}: \pi_{1}(M) \rightarrow \mathrm{GL}(\mathbb{C})$.

2) Для произвольной пары $\omega, \omega^{\prime}$ 1-форм таких, что $\omega-\omega^{\prime}=d \phi, \phi \in C^{\infty}(M), \kappa o$ гомологии $H_{\lambda \omega}^{*}(M, \mathbb{C})$ и $H_{\lambda \omega^{\prime}}^{*}(M, \mathbb{C})$ изоморфны. Этот изоморфизм мохет быть задан при помощи калибровочного преобразования

$$
a \rightarrow e^{\lambda \phi} a, \quad d \rightarrow e^{\lambda \phi} d e^{-\lambda \phi}=d+\lambda d \phi \wedge
$$

Когомологии $H_{\lambda \omega}^{*}(M, \mathbb{C})$ возникают естественным образом в рамках теории Морса-Новикова. В классической теории Морса ставится задача о связи множества критических точек $d f=0$ произвольной функции Морса $f$ на замкнутом компактном многообразии $M$ с топологическими инвариантами многообразия $M$. С. П. Новиков в [1], [2] построил аналог такой теории для замкнутой, но не обязательно точной 1-формы $\omega$, которая является морсовской, т.е. в достаточно малой окрестности произвольной точки $x \in M$ она является дифференциалом некоторой функции Морса $d f$. Нули морсовской формы $\omega$ являются изолированными; значит, можно определить индекс каждого из них, как индекс критической точки локальной функции $f, \omega=d f$. Число нулей формы $\omega$ индекса $p$ обозначим через $m_{p}(\omega)$.

Теорема 1.1 (А.В. Пажитнов [4]). Для достаточно больиих вещественных чисел $\lambda$ имеем следуюшую оценку:

$$
m_{p}(\omega) \geqslant \operatorname{dim} H_{\rho_{\lambda \omega}}^{*}(M, \mathbb{C}) \quad \forall p
$$

2. Точная последовательность Диксмье когомологий алгебры Ли. Пусть $\mathfrak{g}$ - алгебра Ли над полем $\mathbb{K}$ и $\rho: \mathfrak{g} \rightarrow \mathfrak{g l}(V)$ - некоторое ее представление. Обозначим $C^{q}(\mathfrak{g}, V)$ пространство всех $q$-линейных кососимметричных отображений из $\mathfrak{g}$ в $V$. Определен алгебраический комплекс

$$
V=C^{0}(\mathfrak{g}, V) \stackrel{d}{\rightarrow} C^{1}(\mathfrak{g}, V) \stackrel{d}{\rightarrow} C^{2}(\mathfrak{g}, V) \stackrel{d}{\rightarrow} \cdots,
$$

в котором дифференциал $d$ задан следующим образом:

$$
\begin{aligned}
d f\left(X_{1}, \ldots, X_{q+1}\right)= & \sum_{i=1}^{q+1}(-1)^{i+1} \rho\left(X_{i}\right)\left(f\left(X_{1}, \ldots, \widehat{X}_{i}, \ldots, X_{q+1}\right)\right) \\
& +\sum_{1 \leqslant i<j \leqslant q+1}(-1)^{i+j} f\left(\left[X_{i}, X_{j}\right], X_{1}, \ldots, \widehat{X}_{i}, \ldots, \widehat{X}_{j}, \ldots, X_{q+1}\right)
\end{aligned}
$$

Когомологии комплекса $\left(C^{*}(\mathfrak{g}, V), d\right)$ назьваются когомологиями алгебры Ли $\mathfrak{g}$ с коэффициентами в представлении $\rho: \mathfrak{g} \rightarrow \mathfrak{g l}(V)$.

Рассмотрим тривиальное представление $\rho_{0}: \mathfrak{g} \rightarrow \mathbb{K}, \rho_{0}=0$. Когомологии соответствующего комплекса $\left(\Lambda^{*}\left(\mathfrak{g}^{*}\right), d\right)$ назьваются когомологиями с тривиальными коэффичиентами алгебры Ли $\mathfrak{g}$ и обозначаются $H^{*}(\mathfrak{g})$. 
Отметим, что оператор $d: \mathfrak{g}^{*} \rightarrow \Lambda^{2}\left(\mathfrak{g}^{*}\right)$, взятый со знаком минус, является двойственньм отображением к скобке Ли $[\cdot, \cdot]: \Lambda^{2}(\mathfrak{g}) \rightarrow \mathfrak{g}$, а тривиальность оператора $d^{2}$ эквивалентна тождеству Якоби в алгебре Ли g.

Непосредственно из определения следует, что $H^{1}(\mathfrak{g})$ является двойственным пространством к $\mathfrak{g} /[\mathfrak{g}, \mathfrak{g}]$, а значит,

1) $\operatorname{dim} H^{1}(\mathfrak{g}) \geqslant 2$ для нильпотентной алгебры Ли $\mathfrak{g}$;

2) $\operatorname{dim} H^{1}(\mathfrak{g}) \geqslant 1$ для разрешимой алгебры Ли $\mathfrak{g}$;

3) $H^{1}(\mathfrak{g})=0$ для полупростой алгебры Ли $\mathfrak{g}$.

Рассмотрим произвольную алгебру Ли $\mathfrak{g}$ над полем $\mathbb{K}$ с нетривиальной первой группой когомологий $H^{1}(\mathfrak{g})$ и выберем некоторую замкнутую 1-форму $\omega$. Для произвольного $\lambda \in \mathbb{K}$ можно определить:

1) новьй деформированньй дифференциал $d_{\lambda \omega}$ в $\Lambda^{*}\left(\mathfrak{g}^{*}\right)$ при помощи формулы

$$
d_{\lambda \omega}(a)=d a+\lambda \omega \wedge a
$$

2) одномерное представление

$$
\rho_{\lambda \omega}: \mathfrak{g} \rightarrow \mathbb{K}, \quad \rho_{\lambda \omega}(\xi)=\lambda \omega(\xi), \quad \xi \in \mathfrak{g}
$$

УТВЕРЖДЕНИЕ 2.1. Комплекс $\left(\Lambda^{*}\left(\mathfrak{g}^{*}\right), d_{\lambda \omega}\right)$ совпадает с коцепным комплексом алгебры Ли $\mathfrak{g}$ с коэффичиентами в одномерном представлении $\rho_{\lambda \omega}: \mathfrak{g} \rightarrow \mathbb{K}$.

ДОКАЗАТЕЛЬСТвО следует непосредственно из

$$
(\lambda \omega \wedge a)\left(X_{1}, \ldots, X_{q+1}\right)=\sum_{i=1}^{q+1}(-1)^{i+1} \lambda \omega\left(X_{i}\right)\left(a\left(X_{1}, \ldots, \widehat{X}_{i}, \ldots, X_{q+1}\right)\right)
$$

ЗАмЕчАниЕ 2.1. Когомологии $H_{\lambda \omega}^{*}(\mathfrak{g})$ совпадают с когомологиями с тривиальными коэффициентами $H^{*}(\mathfrak{g})$, если $\lambda=0$. Отметим также, что если $\lambda \neq 0$, то деформированный дифференциал $d_{\lambda \omega}$, вообще говоря, не совместим с внешним произведением в $\Lambda^{*}\left(\mathfrak{g}^{*}\right)$ :

$$
d_{\lambda \omega}(a \wedge b)=d(a \wedge b)+\lambda \omega \wedge a \wedge b \neq d_{\lambda \omega}(a) \wedge b+(-1)^{\operatorname{deg} a} a \wedge d_{\lambda \omega}(b)
$$

тем самым, когомологии $H_{\lambda \omega}^{*}(\mathfrak{g})$ не имеют естественной мультипликативной структуры при $\lambda \neq 0$.

Ненулевая замкнутая 1-форма $\omega$ задает идеал $\mathfrak{b}_{\omega}=\{x \in \mathfrak{g}, \omega(x)=0\}$ коразмерности 1 в g. Выберем вектор $X \in \mathfrak{g}$ такой, что $\omega(X)=1$, и рассмотрим сопряженньй оператор $\operatorname{ad} X^{*}: \mathfrak{b}_{\omega}^{*} \rightarrow \mathfrak{b}_{\omega}^{*}$ к оператору ad $X: \mathfrak{b}_{\omega} \rightarrow \mathfrak{b}_{\omega}$. С его помощью мы можем определить дифференцирование $\operatorname{ad} X^{*}$ степени 0 внешней алгебры $\Lambda^{*}\left(\mathfrak{b}_{\omega}^{*}\right)$ :

$$
\operatorname{ad} X^{*}(a \wedge b)=\operatorname{ad} X^{*} a \wedge b+a \wedge \operatorname{ad} X^{*} b \quad \forall a, b \in \Lambda^{*}\left(\mathfrak{b}_{\omega}^{*}\right) .
$$

Дифференцирование $\mathrm{ad} X^{*}$ перестановочно с дифференциалом $d$ и соответствующее отображение в когомологиях $H^{*}\left(\mathfrak{b}_{\omega}\right)$ мы будем обозначать тем же символом $\mathrm{ad} X^{*}$, a тождественное отображение - символом Id соответственно. 
Теорема 2.1 (Диксмье [9]). Существует длинная точная последовательность когомологий алгебр Ли:

$$
\begin{gathered}
\ldots \stackrel{(-1)^{i}\left(\stackrel{\operatorname{ad} X^{*}}{\longrightarrow}-\lambda \mathrm{Id}\right)}{\longrightarrow} H^{i-1}\left(\mathfrak{b}_{\omega}\right) \stackrel{\wedge \omega}{\longrightarrow} H_{\lambda \omega}^{i}(\mathfrak{g}) \stackrel{r_{i}}{\longrightarrow} H^{i}\left(\mathfrak{b}_{\omega}\right) \\
\quad(-1)^{i+1} \stackrel{\left(\operatorname{ad} X^{*}-\lambda \mathrm{Id}\right)}{\longrightarrow} H^{i}\left(\mathfrak{b}_{\omega}\right) \longrightarrow \cdots,
\end{gathered}
$$

$2 \partial e$

1) гомоморфизм $r_{i}: H_{\lambda \omega}^{i}(\mathfrak{g}) \rightarrow H^{i}\left(\mathfrak{b}_{\omega}\right)$ есть гомоморфизм ограничения;

2) $\wedge \omega: H^{*-1}\left(\mathfrak{b}_{\omega}\right) \rightarrow H^{*}(\mathfrak{g})$ порожден внешним умножсением справа на $\omega:$

$$
\xi \rightarrow \xi \wedge \omega
$$

3) гомоморфизмы аd $X^{*}: H^{i}\left(\mathfrak{b}_{\omega}\right) \rightarrow H^{i}\left(\mathfrak{b}_{\omega}\right)$ индуиированы дифферениированием $\operatorname{ad} X^{*}$ степени 0 внешней алгебры $\Lambda^{*}\left(\mathfrak{b}_{\omega}^{*}\right)$.

ДокАЗАТЕльство. Каждая форма $f \in \Lambda^{*}(\mathfrak{g})$ может быть представлена как $f=$ $f^{\prime} \wedge \omega+f^{\prime \prime}$, где $f^{\prime} \in \Lambda^{*-1}\left(\mathfrak{b}_{\omega}^{*}\right)$ и $f^{\prime \prime} \in \Lambda^{*}\left(\mathfrak{b}_{\omega}^{*}\right)$. Вьпишем короткую точную последовательность алгебраических комплексов

$$
0 \longrightarrow \Lambda^{*-1}\left(\mathfrak{b}_{\omega}^{*}\right) \stackrel{\wedge \omega}{\longrightarrow} \Lambda^{*}\left(\mathfrak{g}^{*}\right) \longrightarrow \Lambda^{*}\left(\mathfrak{b}_{\omega}^{*}\right) \longrightarrow 0
$$

Эта короткая точная последовательность комплексов порождает длинную точную последовательность в когомологиях. Свойства гомоморфизмов $r_{i}$ и $\Lambda \omega$ очевидны. Гомоморфизм же $H^{q}\left(\mathfrak{b}_{\omega}\right) \rightarrow H^{q}\left(\mathfrak{b}_{\omega}\right)$ рассмотрим подробнее. Для этого сначала определим отображение

$$
\Lambda^{q+1}\left(\mathfrak{g}^{*}\right) \rightarrow \Lambda^{q}\left(\mathfrak{b}_{\omega}^{*}\right), \quad f \in \Lambda^{q+1}\left(\mathfrak{g}^{*}\right) \rightarrow f_{X} \in \Lambda^{q}\left(\mathfrak{b}_{\omega}^{*}\right),
$$

где $f_{X}\left(X_{1}, \ldots, X_{q}\right)=f\left(X_{1}, \ldots, X_{q}, X\right)$. Тем самым, $f_{X}=f^{\prime}$, если $f=f^{\prime} \wedge \omega+f^{\prime \prime}$, и $(\lambda \omega \wedge f)_{X}=(-1)^{\operatorname{deg} f} \lambda f$.

Верна очевидная формула

$$
\begin{aligned}
(d f)_{X}\left(X_{1}, \ldots, X_{q}\right)= & -\sum_{1 \leqslant j \leqslant q}(-1)^{j+q+1} f\left(\operatorname{ad} X\left(X_{j}\right), X_{1}, \ldots, \widehat{X}_{j}, \ldots, X_{q}\right) \\
& +\sum_{1 \leqslant i<j \leqslant q}(-1)^{i+j} f\left(\left[X_{i}, X_{j}\right], X_{1}, \ldots, \widehat{X}_{i}, \ldots, \widehat{X}_{j}, \ldots, X_{q}, X\right) \\
= & \left((-1)^{q+1} \operatorname{ad} X^{*}(f)+d\left(f_{X}\right)\right)\left(X_{1}, \ldots, X_{q}\right)
\end{aligned}
$$

Следовательно, связывающий гомоморфизм $H^{q}\left(\mathfrak{b}_{\omega}\right) \rightarrow H^{q}\left(\mathfrak{b}_{\omega}\right)$ длинной точной последовательности когомологий совпадает с гомоморфизмом, порожденным оператором $(-1)^{q+1}\left(\operatorname{ad} X^{*}-\lambda \mathrm{Id}\right)$, которьй тем самым не зависит от выбора вектора $X$.

Обозначим множество собственных значений оператора $\operatorname{ad} X^{*}: H^{k}\left(\mathfrak{b}_{\omega}\right) \rightarrow H^{k}\left(\mathfrak{b}_{\omega}\right)$ через $\operatorname{Spec}^{k}(\omega)$. 
СлЕДСтвиЕ 2.1. Пусть $\mathfrak{g}-n$-мерная алгебра Ли $u \omega \in \mathfrak{g}^{*}, \omega \neq 0, d \omega=0$. Тогда

1) когомологии $H_{\lambda \omega}^{*}(\mathfrak{g})$ являются нетривиальныцми тогда и только тогда, когда

$$
\lambda \in \bigcup_{k=1}^{n} \operatorname{Spec}^{k}(\omega)
$$

2) і-е число Бетти $b_{\lambda \omega}^{i}(\mathfrak{g})=\operatorname{dim} H_{\lambda \omega}^{i}(\mathfrak{g})$ равно

$$
b_{\lambda \omega}^{i}(\mathfrak{g})=k_{\lambda \omega}^{i}+k_{\lambda \omega}^{i-1}
$$

əде число $k_{\lambda \omega}^{i}$ есть размерность ядра оператора $\operatorname{ad} X^{*}-\lambda \operatorname{Id}: H^{i}\left(\mathfrak{b}_{\omega}\right) \rightarrow$ $H^{i}\left(\mathfrak{b}_{\omega}\right)$.

ПримеР 2.1. Пусть $\mathfrak{g}$ - алгебра Ли, определенная базисом $X, e_{1}, e_{2}, \ldots, e_{n}$ и коммутационными соотношениями (мы будем в дальнейшем опускать тривиальные соотношения):

$$
\left[X, e_{1}\right]=e_{1}, \quad\left[X, e_{2}\right]=e_{2}, \quad \ldots, \quad\left[X, e_{n}\right]=e_{n} .
$$

Выберем $\omega, \omega_{1}, \ldots, \omega_{n}$ как элементы соответствующего сопряженного базиса в $\mathfrak{g}^{*}$. В частности,

$$
\omega(X)=1, \quad \omega\left(e_{i}\right)=0, \quad i=1, \ldots, n .
$$

Очевидно, что $\omega$ - замкнутая 1-форма и $\operatorname{ad} X^{*}\left(\omega_{i_{1}} \wedge \cdots \wedge \omega_{i_{p}}\right)=p \omega_{i_{1}} \wedge \cdots \wedge \omega_{i_{p}}$. Следовательно, $\operatorname{Spec}^{p}(\omega)=\{p\}$ и для $\lambda=p$ имеется лишь два нетривиальных числа Бетти:

$$
b_{p \omega}^{p+1}(\mathfrak{g})=b_{p \omega}^{p}(\mathfrak{g})=\left(\begin{array}{c}
n \\
p
\end{array}\right) .
$$

СлЕДСтвИЕ 2.2 [10]. Пусть $\mathfrak{g}-$ нильпотентная алгебра Ли. Тогда

$$
\operatorname{dim} H^{p}(\mathfrak{g}) \geqslant 2, \quad p=1, \ldots, n-1 .
$$

ДокАЗАТЕЛЬСтво. Будем доказывать оценки (6) индукцией по размерности нильпотентных алгебр Ли. В частности, мы предполагаем верными следуюшие неравенства:

$$
\operatorname{dim} H^{p}\left(\mathfrak{b}_{\omega}\right) \geqslant 2, \quad \operatorname{dim} H^{p-1}\left(\mathfrak{b}_{\omega}\right) \geqslant 2 .
$$

Оператор ad $X$ является нильпотентным, а значит, нильпотентными будут и операторы $\operatorname{ad} X^{*}: H^{i}\left(\mathfrak{b}_{\omega}\right) \rightarrow H^{i}\left(\mathfrak{b}_{\omega}\right), i=p, p-1$. Тем самым, для размерностей ядер операторов ad $X^{*}$ имеются оценки $k^{p} \geqslant 1, k^{p-1} \geqslant 1$, откудапри $\lambda=0$ согласно следствию 2.1 и следуют требуемые неравенства (6) при $p \geqslant 2$.

Фактически было доказано и другое

СЛЕДСтвиЕ 2.3. Пусть $\mathfrak{g}$ - нильпотентная алгебра Ли. Когомологии $H_{\lambda \omega}^{*}(\mathfrak{g})$ нетривиальны тогда и только тогда, когда $\lambda \omega=0$. 
ЗАМЕчАнИЕ 2.2. В данной работе мы привели только лишь версию точной последовательности Диксмье, приспособленную к нашему специальному случаю 1-мерного представления $\rho_{\lambda \omega}$ (см. [9]).

\section{3. Когомологии разрешимых алгебр Ли.}

ОПРЕДЕЛЕниЕ 3.1. Вешественная разрешимая алгебра Ли g называется вполне разрешимой, если $\forall X \in \mathfrak{g}$ все собственные значения оператора $\operatorname{ad} X$ являются вещественными числами.

Лемма 3.1. Пусть $\mathfrak{g}-n$-мерная комплексная разрешимая алгебра Ли (вещественная вполне разрешимая алгебра Ли) и $b^{1}(\mathfrak{g})=\operatorname{dim} H^{1}(\mathfrak{g})=k$. Тогда существует базис $\omega_{1}, \ldots, \omega_{n}$ в $\mathfrak{g}^{*}$ такой, что

$$
\begin{gathered}
d \omega_{1}=\cdots=d \omega_{k}=0, \quad d \omega_{i}=\alpha_{i} \wedge \omega_{i}+P_{i}\left(\omega_{1}, \ldots, \omega_{i-1}\right), \\
\alpha_{i}=\sum_{l=1}^{k} a_{i l} \omega_{l}, \quad P_{i}\left(\omega_{1}, \ldots, \omega_{i-1}\right) \in \Lambda^{2}\left(\omega_{1}, \ldots, \omega_{i-1}\right), \quad i=k+1, \ldots, n .
\end{gathered}
$$

ДокАЗАТЕЛЬСтво. Согласно теореме Ли о представлении разрешимой алгебры над алгебраически замкнутым полем [13] существует такой базис $e_{k+1}, \ldots, e_{n}$ в коммутанте $[\mathfrak{g}, \mathfrak{g}]$, что линейные подпространства $\left\langle e_{i}, \ldots, e_{n}\right\rangle, i=k+1, \ldots, n$, являются инвариантными относительно представления $\mathrm{ad}: \mathfrak{g} \rightarrow \mathfrak{g l}([\mathfrak{g}, \mathfrak{g}])$. Дополним базис $e_{k+1}, \ldots, e_{n}$ до базиса $e_{1}, \ldots, e_{n}$ всей алгебры Ли g. Легко видеть, что для функционалов $\omega_{1}, \ldots, \omega_{n}$ сопряженного базиса к $e_{1}, \ldots, e_{n}$ справедливы формулы (7).

Определим $\alpha_{1}=\cdots=\alpha_{k}=0$.

ЗАмЕчАниЕ 3.1. Набор 1-форм $\left\{\alpha_{1}, \ldots, \alpha_{n}\right\} \subset \mathfrak{g}^{*}$ имеет следующий инвариантньй смысл: это - набор весов вполне приводимого представления, ассоциированного с присоединенным представлением $\mathrm{ad}: \mathfrak{g} \rightarrow \mathfrak{g l}(\mathfrak{g})$.

Рассмотрим теперь произвольную комплексную разрешимую (или вещественную вполне разрешимую) алгебру Ли g и выберем базис (7) $\omega_{1}, \ldots, \omega_{n}$ в $\mathfrak{g}^{*}$. Обозначим через $\Lambda^{*}$ внешнюю подалгебру в $\Lambda^{*}\left(\omega_{1}, \ldots, \omega_{n}\right)$, порожденную формами $\omega_{1}, \ldots, \omega_{k}$. Определим возрастающую фильтрацию $F$ коцепного комплекса $\left(\Lambda^{*}\left(\mathfrak{g}^{*}\right), d_{\lambda \omega}\right)$, рассматривая $\Lambda^{*}\left(\mathfrak{g}^{*}\right)$ как модуль над $\Lambda^{*}$ :

$$
0 \subset \Lambda^{*} \subset F^{k+1} \subset F^{k+2} \subset F^{k+1, k+2} \subset F^{k+3} \subset F^{k+1, k+3} \subset \cdots \subset F^{k, \ldots, n}=\Lambda^{*}\left(\mathfrak{g}^{*}\right),
$$

где подмодуль $\left\{F^{j_{1}, \ldots, j_{p}}, k<j_{1}<\cdots<j_{p} \leqslant n\right\}$ порожден мономами $\omega_{l_{1}} \wedge \cdots \wedge \omega_{l_{q}}$, $k<l_{1}<\cdots<l_{q}$ такими, что:

1) $l_{q}<j_{p}$

2) $l_{q}=j_{p}, l_{q-1}<j_{p-1}$

3) $l_{q}=j_{p}, l_{q-1}=j_{p-1}, l_{q-2}<j_{p-2}, \ldots$,

и наконец мономами с $l_{q}=j_{p}, \ldots, l_{q-p+1}=j_{1}$. 
Сами подпространства $\left\{F^{j_{1}}, \ldots, j_{p}\right\}$ занумерованы в соответствии с этим лексиграфическим упорядочением. Например,

$$
\begin{gathered}
F^{k+1}=\Lambda^{*} \oplus \Lambda^{*} \wedge \omega_{k+1}, \quad F^{k+2}=\Lambda^{*} \oplus \Lambda^{*} \wedge \omega_{k+1} \oplus \Lambda^{*} \wedge \omega_{k+2}, \\
F^{k+1, k+2}=\Lambda^{*} \oplus \Lambda^{*} \wedge \omega_{k+1} \oplus \Lambda^{*} \wedge \omega_{k+2} \oplus \Lambda^{*} \wedge \omega_{k+1} \wedge \omega_{k+2}, \\
F^{k+3}=\Lambda^{*} \oplus \Lambda^{*} \wedge \omega_{k+1} \oplus \Lambda^{*} \wedge \omega_{k+2} \oplus \Lambda^{*} \wedge \omega_{k+1} \wedge \omega_{k+2} \oplus \Lambda^{*} \wedge \omega_{k+3}, \quad \ldots
\end{gathered}
$$

Фильтрация $F$ совместима с дифференциалом $d+\lambda \omega$, а значит, можно рассмотреть соответствующую спектральную последовательность $E_{r}$. Ее первый член $E_{1}$ изоморфен прямой сумме когомологий комплексов вида $\left(\Lambda^{*} \wedge \omega_{j_{1}} \wedge \cdots \wedge \omega_{j_{p}}, d_{0}\right)$, где дифференциал $d_{0}$ определен следующим образом:

$$
d_{0}\left(\tilde{a} \wedge \omega_{j_{1}} \wedge \cdots \wedge \omega_{j_{p}}\right)=\left(\alpha_{j_{1}}+\cdots+\alpha_{j_{p}}+\lambda \omega\right) \tilde{a} \wedge \omega_{j_{1}} \wedge \cdots \wedge \omega_{j_{p}}
$$

Тем самьм, $H^{*}\left(\Lambda^{*} \wedge \omega_{j_{1}} \wedge \cdots \wedge \omega_{j_{p}}, d_{0}\right)$ тривиальны, если $\alpha_{j_{1}}+\cdots+\alpha_{j_{p}}+\lambda \omega \neq 0$. Выбирая $\lambda \omega$ таким, что

$$
\alpha_{j_{1}}+\cdots+\alpha_{j_{p}}+\lambda \omega \neq 0 \quad \forall j_{1}<\cdots<j_{p}
$$

замечаем, что спектральная последовательность $E_{r}$ вырождается в первом члене $E_{1}$.

Обозначим

$$
\Omega_{\mathfrak{g}}=\left\{\alpha_{i_{1}}+\cdots+\alpha_{i_{p}} \mid 1 \leqslant i_{1}<\cdots<i_{p} \leqslant n, p=1, \ldots, n\right\} .
$$

В случае вешественной разрешимой алгебры Ли g, рассматривая ее комплексификацию, получаем следующую теорему.

ТЕОремА 3.1. Пусть $\mathfrak{g}-$ разрешимая алгебра Ли, $\operatorname{dim} \mathfrak{g}=n, \Omega_{\mathfrak{g}}-$ конечное подмножество в $H^{1}(\mathfrak{g})$, порожденное набором $\left\{\alpha_{1}, \ldots, \alpha_{n}\right\}$ весов вполне приводимого представления, ассоциированного с присоединенным представлением аd. Тогда существует спектральная последовательность $E_{r}$, сходящаяся к когомологиям $H_{\lambda \omega}^{*}(\mathfrak{g})$, первый член $E_{1}$ которой вырождается, если $-\lambda \omega \notin \Omega_{\mathfrak{g}}$.

Отметим, что подмножество $\Omega_{\mathfrak{g}}$ непусто, так как содержит 0 . Вообще говоря, член $E_{1}$ не совпадает с $E_{\infty}$. Поэтому надо ввести подмножество $\widetilde{\Omega}_{\mathfrak{g}} \subset \Omega_{\mathfrak{g}}$ такое, что $E_{\infty} \neq 0$ тогда и только тогда, когда $-\lambda \omega \in \widetilde{\Omega}_{\mathfrak{g}}$.

СлЕДСТВИЕ 3.1. Пусть $\mathfrak{g}$ - разрешимая алгебра Ли. Когомологии $H_{\rho_{\lambda \omega}^{*}}(\mathfrak{g})$ нетривиальны тогда и только тогда, когда - $\omega \in \in \widetilde{\Omega}_{\mathfrak{g}}$ - некоторому непустому конечному подмножеству в $H^{1}(\mathfrak{g})$.

ЗАмЕчАниЕ 3.2. Данное утверждение не следует, вообще говоря, из следствия 2.1

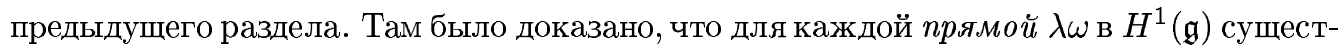
вует лиш конечное число значений параметра $\lambda_{i}$, для которых когомологии $H_{\lambda_{i} \omega}^{*}(\mathfrak{g})$ нетривиальны. 


\section{4. Когомологии солвмногообразий.}

ОПРЕДЕЛЕНИЕ 4.1. Солвмногообразием (нильмногообразием) $M$ назьвается компактное однородное пространство вида $G / \Gamma$, где $G$ является односвязной разрешимой (нильпотентной) группой Ли, а $\Gamma$ - решеткой в $G$.

Приведем вначале несколько примеров нильмногообразий.

ПримеР 4.1. $n$-мерный тор $T^{n}=\mathbb{R}^{n} / \mathbb{Z}^{n}$.

Пример 4.2. Многообразие Гейзенберга $M_{3}=\mathscr{H}_{3} / \Gamma_{3}$, где $\mathscr{H}_{3}$ обозначает группу верхнетреугольных матриц вида

$$
\left(\begin{array}{lll}
1 & x & z \\
0 & 1 & y \\
0 & 0 & 1
\end{array}\right), \quad x, y, z \in \mathbb{R}
$$

a решетка $\Gamma_{3}$ является подгруппой матриц указанного вида с целочисленньми матричными элементами $x, y, z$.

Теорема 4.1 (А.И. Мальцев [14]). Пусть G является односвязной нильпотентой группой Ли, а $\mathfrak{g}$ - ее касательная алгебра Ли. Тогда в $G$ существует кокомпактная решетка $\Gamma$ (иными словами $G / \Gamma$ - компактное пространство), если и только если в алгебре $\mathfrak{g}$ существует базис $e_{1}, e_{2}, \ldots, e_{n}$ такой, что все структурные константы $\left\{c_{i j}^{k}\right\},\left[e_{i}, e_{j}\right]=c_{i j}^{k} e_{k}$, являются рачиональными числами.

Теорема Мальцева дает возможность эффективно строить примеры нильмногообразий. Пусть $\mathfrak{g}$ является нильпотентной алгеброй Ли, заданной при помоши базиса $e_{1}, e_{2}, \ldots, e_{n}$ и коммутационных соотношений $\left[e_{i}, e_{j}\right]=c_{i j}^{k} e_{k}, c_{i j}^{k} \in \mathbb{Q}$. В векторном пространстве $\mathfrak{g}$ с помощью формулы Кэмпбелла-Хаусдорфа [13] определим операцию умножения $*$ :

$$
x * y=x+y+\frac{1}{2}[x, y]+\cdots .
$$

Нильпотентная группа Ли $G=(\mathfrak{g}, *)$ обладает кокомпактной решеткой $\Gamma$ - подгруппой, порожденной элементами базиса $e_{1}, e_{2}, \ldots, e_{n}$, а значит, определено и соответствующее нильмногообразие $G / \Gamma$.

Следующий пример солвмногообразия уже не является нильмногообразием.

ПримеР 4.3. Рассмотрим полупрямое произведение $G_{0}=\mathbb{R} \ltimes \mathbb{R}^{2}$, где $\mathbb{R}$ действует на $\mathbb{R}^{2}$ посредством

$$
t \rightarrow \phi(t)=\left(\begin{array}{cc}
a^{t} & 0 \\
0 & a^{-t}
\end{array}\right)
$$

где $a+a^{-1}=n \in \mathbb{N}, a \neq 1, a>0$. Тогда

$$
\phi(1)=C^{-1}\left(\begin{array}{cc}
0 & 1 \\
-1 & n
\end{array}\right) C
$$

для некоторой матрицы $C \in G L(2, \mathbb{R})$. В этом случае существует решетка $L \subset \mathbb{R}^{2}$, инвариантная относительно оператора $\phi(1)$. Действие $\mathbb{Z} \subset \mathbb{R}$ на $L$ определено оператором $\phi(1)$ и можно определить решетку $\Gamma \subset G$ как полупрямое произведение $\mathbb{Z} \ltimes_{\phi(1)} L$. 
Решетки, отвечающие различньм значениям $n$, неизоморфны, а соответствующие солвмногообразия попарно топологически не эквивалентны.

Отметим также, что солвмногообразие из предыдущего примера является расслоением над $S^{1}$ со слоем $T^{2}$. Этот факт обобщается в следующей теореме Мостова.

ТЕОРема 4.2 [15]. Всякое компактное солвмногообразие расслаивается над тором с некоторым нильмногообразием в качестве слоя.

Из данной теоремы вытекает, что на произвольном солвмногообразии всегда существует 1-форма $\omega$ без нулей.

ОПРЕДЕЛЕНИЕ 4.2. Разрешимая группа Ли $G$ назьвается вполне разрешимой, если ее касательная алгебра Ли g является вполне разрешимой.

Комплекс де Рама $\Lambda^{*}(G / \Gamma)$ солвмногообразия $G / \Gamma$ может быть отождествлен с подкомплексом $\Lambda_{\Gamma}^{*}(G) \subset \Lambda^{*}(G)$ левоинвариантных относительно действия решетки $Г$ дифоференциальных форм на группе $G$. В свою очередь, комплекс $\Lambda_{\Gamma}^{*}(G)$ содержит подкомплекс $\Lambda_{G}^{*}(G)$ левоинвариантных форм относительно уже полного действия всей группы $G$. Последний подкомплекс $\Lambda_{G}^{*}(G)$ изоморфен естественным образом коцепному комплексу $\Lambda^{*}(\mathfrak{g})$ алгебры Ли $\mathfrak{g}$. Рассмотрим определенное таким образом включение

$$
\psi: \Lambda^{*}(\mathfrak{g}) \rightarrow \Lambda^{*}(G / \Gamma)
$$

ТЕоремА 4.3 (Хаттори [10]). Пусть G/Г - компактное солвмногообразие, где $G$ - вполне разрешимая группа Ли. Тогда отображение включения $\psi: \Lambda^{*}(\mathfrak{g}) \rightarrow$ $\Lambda^{*}(G / \Gamma)$ индуиирует изоморфизм $\psi^{*}: H^{*}(\mathfrak{g}) \rightarrow H^{*}(G / \Gamma, \mathbb{R})$ в когомологиях.

ПримеР 4.4. Обозначим через $\mathscr{V}_{n}$ нильпотентную алгебру Ли с базисом $e_{1}, e_{2}, \ldots, e_{n}$ и коммутационными соотношениями

$$
\left[e_{i}, e_{j}\right]= \begin{cases}(j-i) e_{i+j}, & i+j \leqslant n \\ 0, & i+j>n .\end{cases}
$$

Когомологии с локальными коэффициентами нильпотентных алгебр Ли $\mathscr{V}_{n}$ и соответствующих нильмногообразий $M_{n}$ были рассмотрены Алания в [6]. В частности им была доказана тривиальность когомологий $H_{\lambda \omega}^{*}\left(M_{n}, \mathbb{C}\right)$ при $\lambda \omega \neq 0$. В [6] было замечено, что аналогичньй результат справедлив и для произвольного нильмногообразия. Но, как мы показали ранее в следствии 2.3, это следует из более общей теоремы Диксмье [9] о когомологиях нильпотентных алгебр Ли.

ЗАмЕчАниЕ 4.1. Теорема Хаттори обобщает классический результат Номидзу [8] для нильмногообразий. Для произвольного солвмногообразия $G / \Gamma$ отображение $\psi^{*}$ не является изоморфизмом, но остается инъективньм (см. [16]).

Таким образом, для вполне разрешимой групшы Ли $G$ каждый класс $[\omega] \in H^{1}(G / \Gamma, \mathbb{R})$ может быть представлен левоинвариантной (относительно действия $G$ ) 1-формой $\omega$.

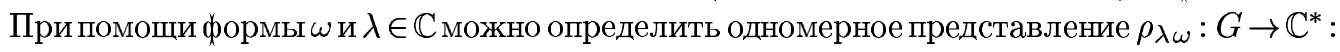

$$
\rho_{\lambda \omega}(g)=\exp \int_{\gamma(e, g)} \lambda \omega
$$


г де $\gamma(e, g)$ - некоторьй путь, соединяющий единицу е с $g$ в группе $G$ (напомним, что $G$ является односвязной). Так как $\omega$ - левоинвариантная 1-форма, справедливо следующее свойство:

$$
\int_{\gamma\left(e, g_{1} g_{2}\right)} \lambda \omega=\int_{\gamma\left(e, g_{1}\right)} \lambda \omega+\int_{\gamma\left(g_{1}, g_{1} g_{2}\right)} \lambda \omega=\int_{\gamma\left(e, g_{1}\right)} \lambda \omega+\int_{g_{1}^{-1} \gamma\left(g_{1}, g_{1} g_{2}\right)} \lambda \omega
$$

а это и значит, что $\rho_{\lambda \omega}\left(g_{1} g_{2}\right)=\rho_{\lambda \omega}\left(g_{1}\right) \rho_{\lambda \omega}\left(g_{2}\right)$. Представление $\rho_{\lambda \omega}$ индуцирует представление соответствующей алгебры Ли $\mathfrak{g}$ (его мы обозначим тем же символом): $\rho_{\lambda \omega}(X)=\lambda \omega(X)$.

Теорема Хаттори, в свою очередь, может быть обобщена на более широкий класс разрешимых групा Ли.

ОПРЕДЕЛЕНИЕ 4.3. Пусть $G$-односвязная грушпа Ли и $\Gamma \subset G$ - еерешетка. Пусть $\rho$ является представлением группы $G$ в конечномерном комплексном векторном пространстве $F$. Символ Ad будет обозначать присоединенное представление $G$ и в ее касательной алгебре Ли $\mathfrak{g}$ и в ее комплексификации $\mathfrak{g}_{\mathbb{C}}$. Будем говорить, что представление $\rho$ является Г-определяемым, если подгруппы $\rho(\Gamma)$ и $\rho(G)$ имеют одинаковое замькание по Зарисскому в группе $\operatorname{Aut}_{\mathbb{C}}(F)$. Представление $\rho$ назьвается $\Gamma$-допустимым, если представление $\rho \oplus \mathrm{Ad}$ является Г-определяемым.

Теорема 4.4 (Мостов [17, теорема 7.26]). Пусть $G$ - односвязная разрешимая группа Ли и ГС - ее решетка. Пусть $\rho$ является конечномерным Г-допустимым представлением $G$ в комплексном векторном пространстве $F$. Тогда включение $\psi: \Lambda^{*}(\mathfrak{g}, F) \rightarrow \Lambda^{*}(G / \Gamma, F)$ комплексов дифференциальных форм со значениями в $F$ индуиирует изоморфизм в когомологиях $\psi^{*}: H_{\rho}^{*}(\mathfrak{g}, F) \rightarrow H_{\rho}^{*}(G / \Gamma, F)$, әде символ $\rho$ обозначает такжсе представление алгебры Ли $\mathfrak{g}$, индуцированное представлением группь $\rho: G \rightarrow \operatorname{Aut}_{\mathbb{C}}(F)$.

В данной работе ограничимся случаем солвмногообразий с вполне разрешимой группой Ли $G$. Из теоремы Хаттори следует следующее важное

СлЕДСТВИЕ 4.1. Пусть $G / \Gamma$ - компактное солвмногообразие, $G$ является вполне разрешимой группой Ли $и \omega$ - замкнутая 1-форма на G/Г. Когомологии $H_{\lambda \omega}^{*}(G / \Gamma, \mathbb{R})$ изоморфны когомологиям алгебры Ли $H_{\lambda \omega^{\prime}}^{*}(\mathfrak{g})$, где $\omega^{\prime} \in \mathfrak{g}^{*}$ является левоинвариантной 1-формой, представляющей класс $[\omega] \in H^{1}(G / \Gamma, \mathbb{R})$.

Следствие 4.1 вместе со следствием 3.1 приводит нас к

Теорема 4.5. Пусть $G / \Gamma$ - компактное солвмногообразие, где $G$ - вполне разрешимая группа Ли и $H_{\lambda \omega}^{*}(G / \Gamma, \mathbb{C})$ являются нетривиальными тогда и только тогда, когда $-\lambda[\omega] \in \widetilde{\Omega}_{\mathfrak{g}}-$ конечному подмножеству в $H^{1}(G / \Gamma, \mathbb{R})$, корректно определенному в терминах соответствующей алгебры Ли g.

СлЕДСТВИЕ 4.2. Пусть $G / \Gamma$ - компактное нильмногообразие. Когомологии $H_{\lambda \omega}^{*}(G / \Gamma, \mathbb{C})$ тривиальны тогда и только тогда, когда $\lambda \omega \neq 0$.

Рассмотрим трехмерное солвмногообразие $M=G_{0} / \Gamma$ из примера 4.3. Соответствующая алгебра Ли g⿻ изоморфна алгебре Ли, заданной при помощи базиса $e_{1}, e_{2}, e_{3}$ и 
коммутационных соотношений

$$
\left[e_{1}, e_{2}\right]=e_{2}, \quad\left[e_{1}, e_{3}\right]=-e_{3} .
$$

Обозначим через $\omega_{1}, \omega_{2}, \omega_{3}$ элементы сопряженного базиса в $\mathfrak{g}^{*}$. Тогда в коцепном комплексе алгебры $\mathfrak{g}$ выполняются следуюшие формулы для его дифференциала:

$$
d \omega_{1}=0, \quad d \omega_{2}=-\omega_{1} \wedge \omega_{2}, \quad d \omega_{3}=\omega_{1} \wedge \omega_{3} .
$$

Таким образом,

$$
\alpha_{1}=0, \quad \alpha_{2}=-\omega_{1}, \quad \alpha_{3}=\omega_{1} .
$$

Легко видеть, что когомологии $H_{\lambda \omega}^{*}\left(\mathfrak{g}_{0}\right)$ нетривиальны тогда и только тогда, когда $\lambda \omega=$ $0, \pm \omega_{1}$. Соответствующие числа Бетти

$$
b_{\lambda \omega}^{p}(M)=\operatorname{dim} H_{\lambda \omega}^{p}\left(G_{0} / \Gamma\right)=\operatorname{dim} H_{\lambda \omega}^{p}\left(\mathfrak{g}_{0}\right)
$$

солвмногообразия $M=G_{0} / \Gamma$ равны

$$
\begin{gathered}
b_{ \pm \omega_{1}}^{0}(M)=0, \quad b_{ \pm \omega_{1}}^{1}(M)=b_{ \pm \omega_{1}}^{2}(M)=1, \quad b_{ \pm \omega_{1}}^{3}(M)=0 \\
b^{0}(M)=b^{1}(M)=b^{2}(M)=b^{3}(M)=1
\end{gathered}
$$

\section{СПИСОК ЦИТИРОВАННОЙ ЛИТЕРАТУРЫ}

[1] Новиков С. П. Многозначные функции и функционалы. Аналог теории Морса // Докл. АН CCCP. 1981. T. 260. №1. C. 31-35.

[2] Новиков С. П. Гамильтонов формализм и многозначный аналог теории Морса // УМН. 1982. T. 37. № 5. С. 3-49.

[3] Новиков С. П. Блоховские гомологии. Критические точки функций и замкнутых 1-форм // Докл. АН СССР. 1986. Т. 287. №6. С. 1321-1324.

[4] Пажитнов А. В. Аналитическое доказательство вещественной части неравенств Новикова // Докл. АН СССР. 1987. Т. 293. №6. С. 1305-1307.

[5] Witten E. Supersymmetry and Morse theory // J. Differential Geom. 1982. V. 17. P. 661-692.

[6] Алания Л. А. Когомологии с локальными коэффициентами некоторых нильмногообразий // УМН. 1999. Т. 54. № 5. С. 147-148.

[7] Миллионщиков Д. В. Когомологии нильмногообразий и теорема Гончаровой // УМН. 2001. Т. 56. № 4. С. 153-154.

[8] Nomizu K. On the cohomology of homogeneous spaces of nilpotent Lie groups // Ann. of Math. 1954. V. 59. P. 531-538.

[9] Dixmier J. Cohomologie des algebres de Lie nilpotentes // Acta Sci. Math. (Szeged). 1955. V. 16. P. $246-250$.

[10] Hattori A. Spectral sequence in the deRham cohomology of fibre bundles // J. Fac. Sci. Univ. Tokyo. Sect. 1. 1960. V. 8. № 4. P. 289-331.

[11] Миллионщиков Д. В. Когомологии с локальными коэффициентами солвмногообразий и задачи теории Морса-Новикова // УМН. 2002. Т. 57. № 4. С. 183-184.

[12] Стинрод Н. Топология косых произведений. М.: ИЛ, 1953.

[13] Серр ЖК.-П. Алгебры Ли и группы Ли. М.: Мир, 1969.

[14] Мальцев А. И. Об одном классе однородных пространств // Изв. АН СССР. Сер. матем. 1949. T. 13. № 1. C. 9-32.

[15] Mostow G. D. Factor spaces of solvable groups // Ann. of Math. 1954. V. 60. P. 1-27.

[16] Рагунатан М. Дискретные подгруппы групп Ли. М.: Мир, 1977.

[17] Mostow G. D. Cohomology of topological groups and solvmanifolds // Ann. of Math. 1961. V. 73. P. $20-48$. 